\title{
Optimal Control of Nonlinear System for Generator Bidding in Deregulated Power Markets
}

\author{
Youfei Liu, Felix F. Wu, Fellow, IEEE, and K L Teo, Senior Member IEEE
}

\begin{abstract}
In this paper, considering generator's long-term optimization behavior, the generator bidding problem is studied using optimal control theory. In particular, the system demand is treated as a periodic function, and the competition process is then modeled as a dynamic, nonlinear and feedback system with periodic parameters, where the publicly known market clearing price (MCP) is the system output and the feedback signal, and supplier's outputs are the state variables. A software package MSIER3 for numerically solving the general optimal control problem is used for simulation. The performance of the optimal control is investigated, and a sensitivity analysis of system parameters is done through simulation.

Index terms - Power markets, Nonlinear system with periodic parameters, Optimal control, Sensitivity analysis
\end{abstract}

\section{INTRODUCTION}

$\mathbf{I}_{\mathrm{d}}^{\mathrm{N}}$ $\mathrm{N}$ deregulated power markets, the generation dispatching is determined by market mechanism rather than the centralized optimization. It is well known that, duo to the market barriers of long period construction and huge capital investment, the deregulated markets are usually oligopoly, and individual supplier (generator) holds some market power and can manipulate the market price in some extent through the strategic behaviors. In recent years, the issues on how to optimally exploit and utilize the market power either explicitly or implicitly (which is also called as the problem of strategic bidding or optimal bidding) are widely addressed. Lots of work has been reported, and many optimal algorithms have been applied, such as the discrete stochastic optimization trough Markov decision process ${ }^{[1]}$, the stochastic optimization with gene algorithm and Monte Carlo simulation ${ }^{[2]}$, the ordinal optimization ${ }^{[3]}$, the Largrangian relaxation and stochastic dynamic programming ${ }^{[4]}$, and etc. On the other hand, the generator bidding can be modeled as a supplier game, and the game-theory based methods have been widely applied to study generators' strategic behaviors and analyze the Nash equilibrium of deregulated power markets, such as ${ }^{[5][6][7]}$, and etc.

However, they all consider the hourly markets as independent, i.e., bidding is based on myopic behaviors or short-term maximization. The system demand has more or less predictable daily variation. Such temporal effect makes the market dynamic and therefore viewing the

Youfei Liu is with the EEE Department, Hong Kong University, Hong Kong.(e-mail: youfei@eee.hku.hk)

Felix.F Wu is with the EEE Department, Hong Kong University, Hong Kong.(e-mail: ffwu@eee.hku.hk)

K L Teo is with the Applied Mathematics department, Hong Kong Polytechnic University, Hong Kong (email: mateokl @ polyu.edu.hk) competition process as a dynamic feedback system provides a superior model.

In this paper, we formulate the generator bidding problem in deregulated power markets using optimal control theory. The system demand is treated as a periodic function, and the competition process is then modeled as a dynamic nonlinear system with periodic parameters, where the publicly known market clearing price is the system output and the system feedback signal, and supplier's outputs are the state variables. A software package MSIER3 for numerically solving the general optimal control problem is used for the simulation. The performance of the optimal control is investigated. Also a sensitivity of analysis of system parameters is done through simulation, and some interesting findings are given.

The paper is organized as follows. In section II, the generator bidding process in deregulated power markets is modeled as a dynamic, nonlinear and feedback system with periodic parameters. Then in section III the general idea of optimal control application is presented. The numerical simulation and the sensitivity analysis are given in section IV with conclusions in section $\mathrm{V}$.

\section{A DYNAMIC, NONLINEAR AND FEEDBACK SYSTEM WITH PERIODIC PARAMETERS}

In this section, the generator bidding process will be modeled as a dynamic, nonlinear and feedback system with periodic parameters. At first, some assumptions are needed for our explicit mathematical formulation.

\subsection{Assumption of supplier's cost function}

The supplier's cost function is assumed to be quadratic:

$$
\operatorname{Cost}_{i}\left(q_{i}\right)=a_{i}+b_{i} q_{i}+\frac{1}{2} c_{i} q_{i}^{2}, \quad i=1, \cdots, n
$$

Where the coefficients $\left(a_{i}, b_{i}, c_{i}\right)$ are all positive.

\subsection{Periodic system demand}

The system hourly demand function is assumed to be linear:

$$
D(t)=\hat{a}(t)-\hat{b}(t) p(t)
$$

For the demand function is down-sloping, thus $\hat{b}(t)>0$. The corresponding inverse demand function is:

$$
p(t)=e(t)-f(t) D(t)
$$

Where $e(t)=\frac{\hat{a}(t)}{\hat{b}(t)}$ and $f(t)=\frac{1}{\hat{b}(t)}$.

The system demand varies across hours of a day. Over different days, there is notable periodicity. For example, the demand for a specified hour of a day is almost the 
same with the one of the same hour in another day. Suppose that this period is $T$ (Generally $T$ is 24 hour), we have:

$$
\left\{\begin{array} { l } 
{ \hat { a } ( t ) = \hat { a } ( t + T ) } \\
{ \hat { b } ( t ) = \hat { b } ( t + T ) }
\end{array} \text { and } \left\{\begin{array}{l}
e(t)=e(t+T) \\
f(t)=f(t+T)
\end{array}\right.\right.
$$

For the non-storability of power energy, the market balancing condition is:

$$
D(t)=\sum_{i=1}^{n} q_{i}(t)
$$

\subsection{A Dynamic adjustment process}

In the hourly bidding electricity spot markets, the suppliers (generators) submit their hourly bids for generation dispatching to the ISO (Independent System Operator), then based on the submitted bids and the demand function, ISO will determine the MCP (market clearing price) and the scheduled generation for individual supplier ${ }^{[8]}$.

After the market is cleared, individual supplier knows the publicized MCP and his scheduled generation, then in the next round of bidding (hourly-based bid), based on above information, he will adjust his generation bid to maximize the profits. Therefore, the bidding process can be modeled as a dynamic feedback system, where the feedback signal is the MCP. Figure 1 shows the general idea of such dynamic feedback system:

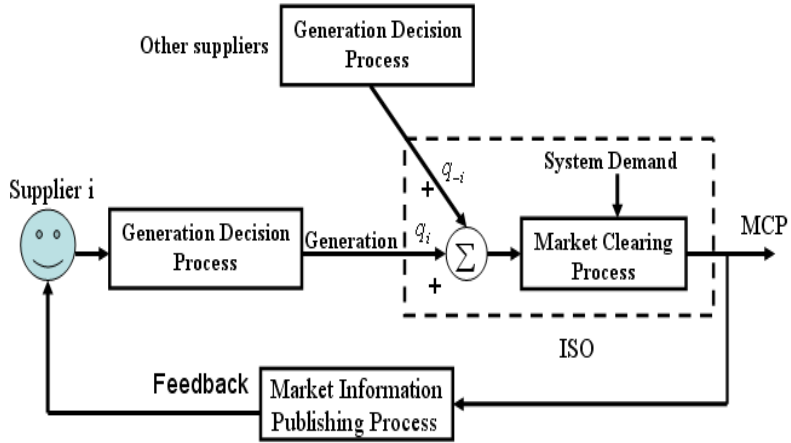

Figure 1 Diagram of dynamic feedback system

In figure 1 , there are three boxes, where the generation decision box is for supplier to make the generation bid decision, and the market clearing box is to clear the market and determine the market clearing price (MCP) which is to balance the total supply and the system demand, the market information box means to publish the market clearing results (such as the MCP and the individual dispatched generation).

Note that the box of generation decision process can be very complex for various decision strategies can be used. For the current study, a dynamic nonlinear adjustment process based on the first order condition for optimality will be proposed in the following.

Supplier's hourly profit function is given as the difference between the revenue and the cost:

$$
\pi_{i}=p q_{i}-\operatorname{Cost}_{i}\left(q_{i}\right)
$$

Then from the first order condition for optimality of equation (6), it is rational to assume that supplier's dynamic response can be described by:

$$
\frac{d q_{i}(t)}{d t}=\lambda_{i} \frac{d \pi_{i}(t)}{d q_{i}(t)}, \lambda_{i}>0
$$

Where $\lambda_{i}$ is a factor representing the speed of adjustment or the preference of adjustment intensity of supplier $i$. Equation (7) also indicates that suppliers will adjust their hourly outputs in the direction of profit increasing.

Through the help of the well-known Conjectural Variation $(\mathrm{CV})$ model in game theory ${ }^{[9][10]}$, together with (1) and (3), equation (7) can be written as:

$$
\begin{aligned}
& \dot{q}_{i}(t)=\lambda_{i} \frac{d \pi_{i}(t)}{d q_{i}(t)}=\lambda_{i}\left(p(t)+\frac{d p}{d q_{i}} q_{i}(t)-\left(b_{i}+c_{i} q_{i}(t)\right)\right) \\
& =\lambda_{i}\left(p(t)-f\left(1+\frac{d \sum_{j \neq i} q_{j}}{d q_{i}}\right) q_{i}(t)-\left(b_{i}+c_{i} q_{i}(t)\right)\right) \\
& =\lambda_{i}\left(p(t)-f\left(1+C V_{i}(t)\right) q_{i}(t)-\left(b_{i}+c_{i} q_{i}(t)\right)\right)
\end{aligned}
$$

And the corresponding difference equation: $q_{i}[t+1]=\lambda_{i}\left(p[t]-f[t]\left(1+C V_{i}[t+1]\right) q_{i}[t]-\left(b_{i}+c_{i} q_{i}[t]\right)\right)+q_{i}[t]$ $=\left(1-\lambda_{i}\left(2 f[t]+f[t] C V_{i}[t+1]+c_{i}\right)\right) q_{i}[t]+\lambda_{i}\left(e[t]-f[t] \sum_{j \neq i} q_{j}[t]-b_{i}\right)(9)$ Where $C V_{i}=\frac{d \sum_{j \neq i} q_{j}[t]}{d q_{i}}$ is called 'conjecture variation', which is the effect on the total quantity output by all other players caused by the change of supplier $i$ 's change. The definition would suggest that $C V_{i}[t+1]=\frac{\sum_{j \neq i} q_{j}[t]-\sum_{j \neq i} q_{j}[t-1]}{q_{i}[t]-q_{i}[t-1]}$.But this would cause numerical problem when $\left(q_{i}[t]-q_{i}[t-1]\right)$ is close to zero as the limit is achieved. In reality, CV is the player's guess of its effect on others. We could model the player's guess of $\mathrm{CV}$ is updated by observing the error term between $C V_{i}[t]$ and $\frac{\sum_{j \neq i} q_{j}[t]-\sum_{j \neq i} q_{j}[t-1]}{q_{i}[t]-q_{i}[t-1]}$, i.e.: $C V_{i}[t+1]$

$=\alpha_{i} C V_{i}[t]+\beta_{i}\left(\sum_{j \neq i} q_{j}[t]-\sum_{j \neq i} q_{j}[t-1]-C V_{i}[t]\left(q_{i}[t]-q_{i}[t-1]\right)\right)(10)$

Where $\alpha_{i}\left(0 \leq \alpha_{i} \leq 1\right)$ is a decay factor, and $\beta_{i}\left(\beta_{i}>0\right)$ an updating factor; $\sum_{j \neq i} q_{j}[t]-\sum_{j \neq i} q_{j}[t-1]$ is the true output adjustment of rival suppliers between two consecutive times, while $C V_{i}[t]\left(q_{i}[t]-q_{i}[t-1]\right)$ is the expected output adjustment.

Note that the advantage of above formulation is that only the information of total dispatched quantity $Q$ is needed, for $\left(\sum_{j \neq i} q_{j}[t]-\sum_{j \neq i} q_{j}[t-1]\right.$ can be derived as 
$(Q[t]-Q[t-1])-\left(q_{i}[t]-q_{i}[t-1]\right)$, or only the information of market price $p$ is needed, for we have $Q[t]=D[t]=\hat{a}[t]-\hat{b}[t] p[t]$. Moreover, the above model has a better property of stability than other CV models. Further study on the stability of above model is out of this paper scope, and will not be addressed here.

\section{FORMULATION OF OPTIMAL CONTROL}

Although with (9), the bidding process in deregulated power markets are modeled as a dynamic, nonlinear and feedback system, the inherited behavior is still myopic, i.e., the individual supplier only makes the short-term optimization or only concerns the instantaneous profits and the impact of current decision on the future profits is ignored. For rationality, the long term optimization over a planning period should be considered. It is well-known that in a dynamic system, the natural way to do the longterm optimization is the optimal control. Unfortunately, no prior literature work has been reported.

Aiming for this, this paper presents a pioneer work to investigate the application of optimal control and study its performance. Without loss of generality, assume that there is a smart supplier (supplier $n$ ) who will adopt the optimal control strategy for maximizing the aggregate profits over a long period time, while other suppliers still make the short-term optimization and will follow the dynamic adjustment process (9). Moreover, assume that the supplier with optimal control has a perfect estimation of the rivals' adjustment processes. Figure 2 shows the general idea about the application of optimal control for the generator bidding in deregulated power markets.

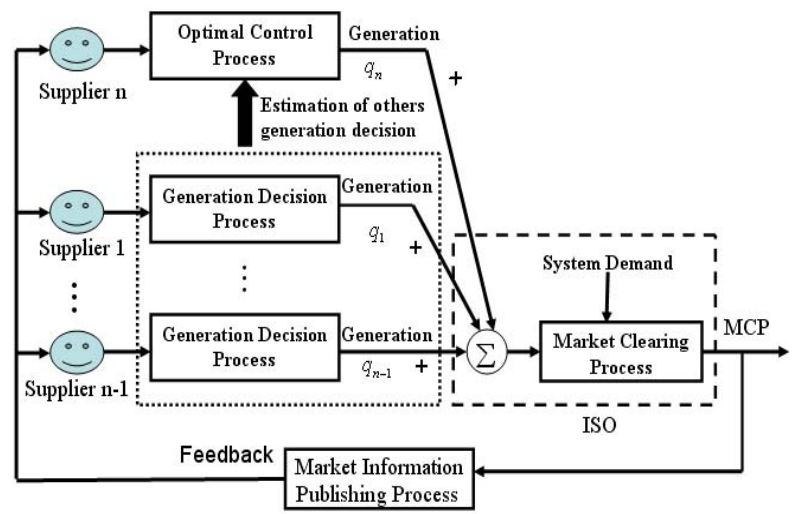

Figure 2 Diagram of optimal control application for the generator bidding

To formulate an optimal control problem for the generator bidding in power markets, we need the objective function, the system state equation and the system output equation, given in follows.

\section{(1)Objective function:}

Supplier $n$ makes the long-term optimization and the objective is:

$$
\max _{q_{n}[t]} \sum_{t=1}^{N T}\left(p[t] q_{n}[t]-\left(a_{n}+b_{n} q_{n}[t]+\frac{1}{2} c_{n} q_{n}[t]^{2}\right)\right)
$$

\section{(2)System state equation:}

The estimation of rivals' generation decision process:

$$
\begin{aligned}
q_{-n}[t+1]= & \lambda_{-n}\left(p[t]-f[t]\left(1+C V_{-n}[t+1]\right) q_{-n}[t]-\left(b_{-n}+c_{-n} q_{-n}[t]\right)\right) \\
& +q_{-n}[t]
\end{aligned}
$$

Where

$$
\begin{aligned}
C V_{-n}[t+1] & =\alpha_{-n} C V_{-n}[t] \\
& +\beta_{-n}\left(q_{n}[t]-q_{n}[t-1]-C V_{-n}[t]\left(q_{-n}[t]-q_{-n}[t-1]\right)\right)
\end{aligned}
$$

Note that the subscript $(-n)$ means supplier $n$ 's aggregated rival.

\section{(3)System output equation:}

$$
p[t]=e[t]-f[t] D[t]=e[t]-f[t]\left(q_{n}[t]+q_{-n}[t]\right)
$$

\section{(4) Constraints on control variable:}

The generation capacity constraints:

$$
q_{n, \min } \leq q_{n}[t] \leq q_{n, \max }
$$

Above equation (11), (12), (13) and (14) consist of our optimal control formulation. For an optimal control problem, the Pontryagin maximum principle will give the necessary conditions for optimality ${ }^{[11]}$. However, for the above optimal control problem in a nonlinear system, it seems impossible to obtain the analytical solution of optimal control rule. Fortunately, there is a unified approach to numerically solve the optimal control problem with all kinds of constraints ${ }^{[11]}$, and a software package MISER3 has come out and can be used to numerically solve the above optimal control problem.

\section{NUMERICAL RESULTS}

The cost function of market supplier is assumed to be:

$$
\operatorname{Cost}(q)=10+1.5 q+\frac{1}{2} 0.001 q^{2}
$$

To demonstrate the advantage of optimal control, the California power real load data is used in the simulations. The following figures show the real unconstrained demand data of California power market on $16^{\text {th }} \sim 20^{\text {th }}$ April 1998.

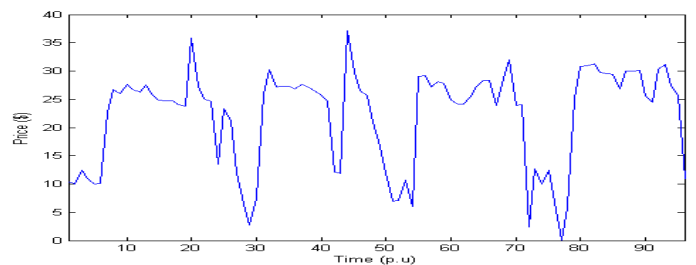

Figure 3 Unconstrained market price on $16^{\text {th }}$, April 1998

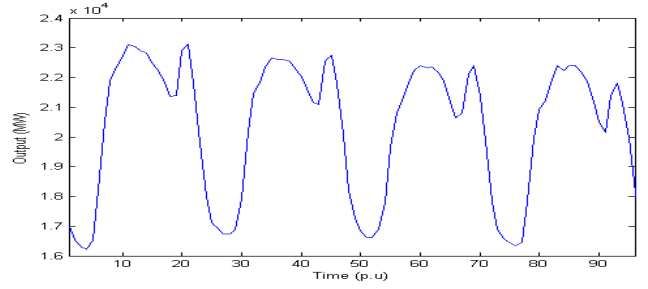

Figure 4 Unconstrained scheduled quantity

From above figures, it is clear that there system demand has a notable periodicity. Generally, the system demand can be modeled by a linear function with periodic parameters (i.e.,equation(2)).The periodic function 
requires that $\hat{a}[t]=\hat{a}[t+24]$ and $\hat{b}[t]=\hat{b}[t+24]$. With the data in above figures, the demand function can be calculated, also the inverse demand function (3). The value of parameter $(\hat{a}, \hat{b})$ in a period is given here:

$\hat{a}=\{36689,42909,46535,45699,40674,33125,36803$, 39367, 40452, 41704, 42249, 42309, 42430,42633, 42460, 42136, 40625, 39490, 39944, 42260, 42928, 41167 $37473,37643\}$;

$\hat{b}=\{1062,1781,2204,2114,1537,621,647,685,709$, $723,704,687,705,719,731,749,734,721,735,724$ $733,747,672,1098\}$. The value of inverse demand function parameter $(e, f)$ can be easily calculated and not given here.

\section{(1) Results from CV competition process}

For simplicity, assume there are two symmetrical suppliers in the market and both of them will follow the $\mathrm{CV}$ competition process (9) With the initial condition $C V_{i}(0)=0 \quad(i=1,2) \quad, \quad\left(q_{1}(0)=8711, q_{2}(0)=8701\right) \quad$, the parameters $\left(\lambda_{i}=60, \alpha_{i}=0.9, \beta_{i}=0.0001\right)(i=1,2)$, the cost function (15) and the above demand function, equation (9) is used for forward iteration to obtain the suppliers' outputs and the market clearing price. For the system parameters are periodic, it is not surprising that the periodic solution of suppliers' outputs is repeated after a short time. To save the space, the details are not given.

Then suppliers' profits can be obtained with equation (6). And supplier 1's aggregate profits in a steady period is given as $\pi_{1}^{C V}=4432200(\$)$.

\section{(2) Results from optimal control}

Now assume that supplier 2 still follows the CV competition process (9) with the parameter given above, while supplier 1 adopts the optimal control with a long planning period (such as 216 hours). MISER3 is used to obtain the suppliers' outputs. Figure 5 shows the suppliers' output trajectories:

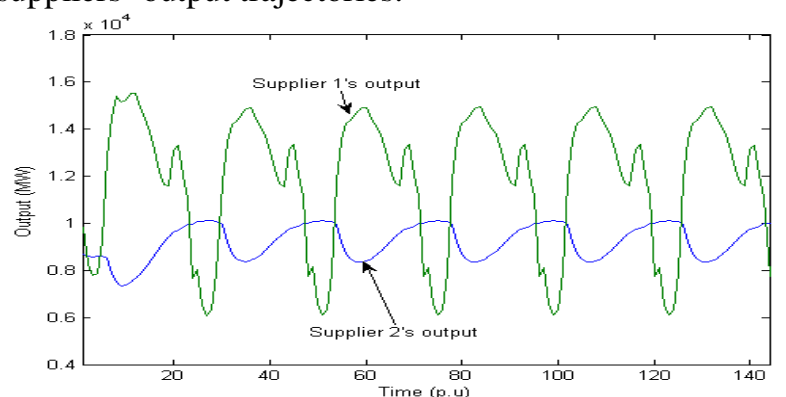

Figure 5 Trajectory of suppliers' outputs: (Supplier 1--optimal control, Supplier 2--CV)

It is also interesting to find that after a period, the periodic solution of suppliers' outputs is repeated.

With the above generation outputs and the above inverse demand function, the market clearing price $p$ can be calculated, and then the suppliers' profits. Now with supplier 1 adopting the optimal control and supplier 2 following the CV process (9), supplier 1's aggregate profit in a steady period is given as $\pi_{1}^{O C}=4606800(\$)$. Compared the results from $\mathrm{CV}$ process, the profit increase is $\Delta \pi_{1}=\pi_{1}^{O C}-\pi_{1}^{C V}=174600(\$)$, and the relative percent is $3.94 \%(4606800-4432200) / 4432200=3.94 \%)$.It is found that optimal control has a better performance over the $\mathrm{CV}$ process. This result is not surprising, for the one with optimal control makes the long term optimization.

\section{(3) Sensitivity analysis of optimal control}

It is easy to understand that the system parameters, such as the demand function coefficients $(\hat{a}, \hat{b})$, the production cost function and the number of market suppliers, will influence the performance of optimal control (i.e., $\Delta \pi_{1}=\pi_{1}^{O C}-\pi_{1}^{C V}$ and $\frac{\left(\pi_{1}^{O C}-\pi_{1}^{C V}\right)}{\pi_{1}^{C V}}$ ). To demonstrate such kind of influences caused by the variation of system parameters, one way is to do the sensitivity analysis through simulation. In what follows, the sensitivity analysis of demand function coefficients (i.e., $(\hat{a}, \hat{b})$ ), marginal cost function slope (i.e., $\left.c_{i}\right)$, and the number of market suppliers (i.e., $n$ ) is given. Doing so, the respective parameter is scaled up or down with other parameters unchanged, and then with the simulation by MISER3, the corresponding suppliers' outputs can be obtained. After that, supplier 1's aggregate profits in a steady period (i.e., $\pi_{1}^{O C}$ ) can be calculated, and then compare the results with the corresponding one (i.e., $\pi_{1}^{C V}$ ) from $\mathrm{CV}$ process, the percent of profit increase (i.e., $\left.\frac{\left(\pi_{1}^{O C}-\pi_{1}^{C V}\right)}{\pi_{1}^{C V}}\right)$ and profits difference (i.e., $\Delta \pi_{1}=\pi_{1}^{O C}-\pi_{1}^{C V}$ ). Figure 6 shows the percent of relative profit increase w.r.t. the system parameter respectively.

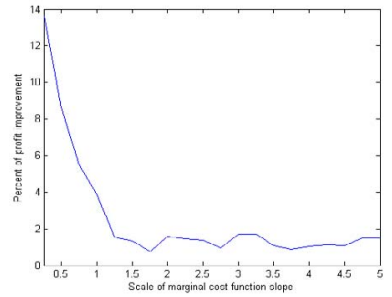

(a)

(c)

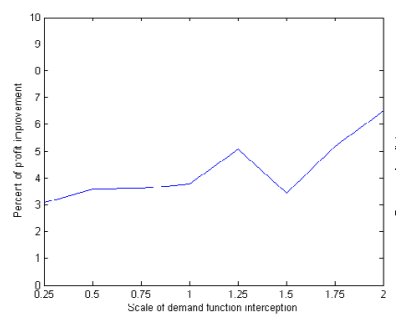

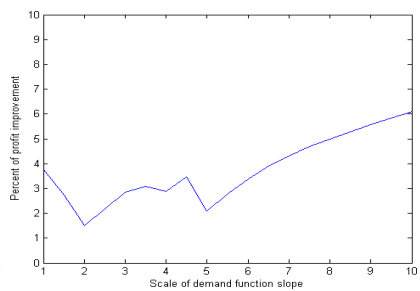

(b)

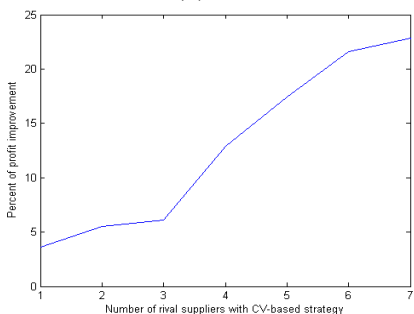

(d)
Figure 6 Percent of profit increase w.r.t. scale of: (a) marginal cost function slope; (b) demand function slope; (c) demand function interception; (d) number of suppliers with $\mathrm{CV}$ competition process. 
Figure 7 shows the profit difference wr.t system parameters respectively.

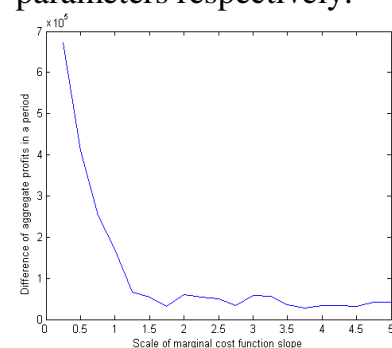

(a)

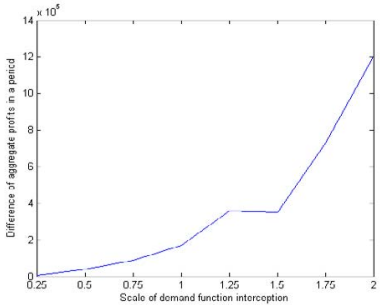

(c)

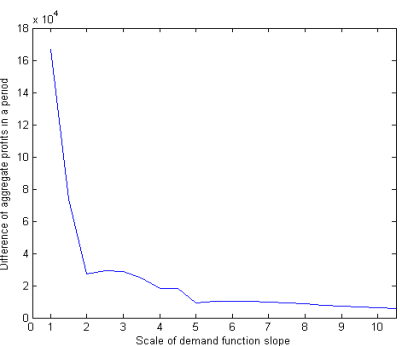

(b)

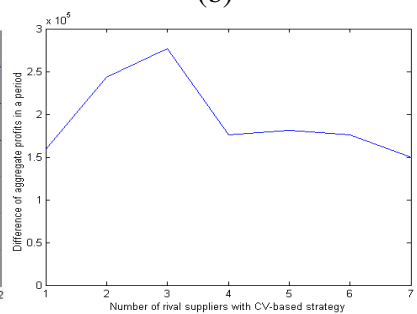

(d)
Figure 7 Profit difference w.r.t. scale of: (a) marginal cost function slope; (b) demand function slope; (c) demand function interception; (d) number of suppliers with $\mathrm{CV}$ competition process.

The percent of profit increase and the profit difference measure the superiority of optimal control. If both of them are rather small, we say that the performance of optima control is not good, otherwise, the performance is good. From the above simulation results, we can conclude that:

(1) With the marginal cost function slope scaling up, the superiority of optimal control will deteriorate, i.e., more expensive the generation production, less beneficial the optimal control

(2) With the demand function slope scaling up or more elastic the system demand, the superiority of optimal control will deteriorate, which means that more elastic the demand, less beneficial the optimal control.

(3) With the demand function interception scaling up, more better the performance of optimal control, which means that more system demand, more beneficial to apply the optimal control.

(4) More suppliers in the market using CV competition process, more better performance the optimal control, which means that more rival suppliers, more beneficial to apply the optimal control.

By the way, it should be pointed out that that supplier 2 who always follows the CV competition process (9) will suffer the profit loss, after supplier 1 switches to the optimal control from the CV process (9).

\section{CONCLUSIONS}

Focusing on the market dynamics and suppliers (generator) long-term optimization behavior, this paper presents a pioneer study about the generator bidding in deregulated power markets using optimal control. In particular, due to the periodic variation of system demand, the generator bidding process is modeled as a dynamic, nonlinear and feedback system. Assuming that there is one smart supplier who will make the long-term optimization, and taking other suppliers' outputs as system state variables and the market clearing price as the system output, an optimal control problem is formulated. Through the help of a software package MISER3, the simulation is done and a sensitivity analysis is given to investigate the performance of optimal control. Some interesting findings are given. The work of this paper can shed lights for the further investigations.

\section{REFERENCES}

[1] H. Song, C.-C. Liu, J. Lawarrée, and R. W. Dahlgren, "Optimal electricity supply bidding by Markov decision process," IEEE Trans. Power Syst., Vol. 15, pp. 618-624, May 2000.

[2] F. Wen and A. K. David, "Optimal bidding strategies and modeling of imperfect information among competitive generators", IEEE Trans. Power Syst., Vol.16, pp. 15-21, Feb. 2001.

[3] X. Guan, Y.-C. Ho, and F. Lai, "An ordinal optimization based bidding strategy for electric power suppliers in the daily energy market", IEEE Trans. Power Syst., Vol.16, pp. 788-797, Nov. 2001.

[4] Ni E., Luh P.B., Rourke S, “Optimal Integrated Generation Bidding and Scheduling With Risk Management Under a Deregulated Power Market", IEEE Trans. Power Syst.,Vol.19, No.1, pp.600-609, 2004.

[5] H. Song, C.-C. Liu, and J. Lawarrée, "Nash equilibrium bidding strategies in a bilateral electricity market", IEEE Trans. Power Syst., vol. 17,pp. 73-79, Feb. 2002.

[6] Jong-Bae Park, Kim B.H., Jin-Ho Kim, Man-Ho Jung, Jong-Keun Park, "A continuous strategy game for power transactions analysis in competitive electricity markets", IEEE Trans. Power Syst., Vol.16, No.4, pp.847-855, 2001.

[7] Ross baldick, Ryan Grant, Edward Kahn, "Linear supply function equilibrium: generalizations, applications, and limitations", UCEI working paper, august, 2000, http://www.ucei.berkeley.edu/PDF/pwp078.pdf.

[8] Mohammad Shahidehpour, Muwaffaq Almoush, Restructured Electrical Power systems: Operation, Trading, and Volatility (MARCEL DEKKER, INC, New York, 2001).

[9] Perry M.K, "Oligopoly and consistent Conjectural variations", Bell journal of Economics, Vol.13, pp.197-205, 1982.

[10] Bresnahan T.F, "Duopoly Models with Consistent Conjectures.", American Economic Review, Vol. 71, pp.934-945,1981

[11] K L Teo, C J Goh, K H Wong, "A unified computational approach to optimal control problems", Longman Scientific \& Technical, 1991.

\section{BIOGRAPHIES}

Youfei Liu received the B.S. and M.S. degrees in control theory and control engineering from University of Science and Technology of China (USTC) in 1999 and 2002, respectively. Now he is a PHD candidate in EEE Dept., Hong Kong University. His current research interest is the analysis of dynamics and stability of power market.

Felix F.Wu received his Ph.D. degree from University of California at Berkeley (UCB). He is now a Chair Professor of EEE dept., Hong Kong University. Prior to that he was a Professor and Vice-Chair of Department of EECS, UCB. His research interests are electric energy industry restructuring, power system investment planning, design of modern control centers, distributed processing etc. 